\title{
Endovascular Treatment of Intracranial Aneurysms with Electrolytically Detachable Platinum Coils
}

Colin Foley, MSc, MD '98

Faculty of Medicine, Dalhousie University, Halifax, Nova Scotia

\begin{abstract}
The incidence of intracranial aneurysms has been estimated to be one to eight per cent of the general population. The most common complication of intracranial aneurysms is rupture with subsequent subarachnoid hemorrhage (SAH), resulting in significant morbidity and mortality. The definitive treatment for SAH in the past has been neurosurgical clipping of the aneurysms to prevent rebleeding. With the development of endovascular techniques in the seventies came an alternative to surgery for patients whose aneurysms were not favorable for clipping and for patients not medically stable enough to tolerate surgery. Of the endovascular techniques, embolization through the use of electrolytically detachable platinum coils appears to be the most promising. This procedure utilizes the electrochemical principles of electrolysis and electrothrombosis to facilitate selec-tive occlusion of intracranial aneurysms while sparing circulation in the parent artery. Evidence of the efficacy of these coils in ruptured intracranial aneurysms has been established in a large multicentered trial. Further investigation, however, is needed to determine the role this endovascular technique will play in first and second line treatment for both ruptured and unruptured intracranial aneurysms.
\end{abstract}

\section{INTRODUCTION}

On the basis of autopsy and angiographic series, it is has been suggested that roughly one to eight per cent of the general population harbor intracranial aneurysms (1). The frequency with which aneurysms are detected is also known to increase with age (2). These are worrisome statistics for health care providers, as the presenta-tion to medical attention of most aneurysms will be dramatic and, unfortunately, devastating to the individual. Many of these patients will be relatively young (mean age is 50 years) (3) and previously healthy when the complications of aneurysm become apparent. Although the pa-tient may experience neurological symptoms secondary to a mass effect of the enlarged aneurysmal blood vessel prior to rupture, the most common complication of intracranial aneu-rysm is bleeding into the subarachnoid space or cerebral tissue from aneurysmal rupture. It has been estimated that 25-28,000 North Americans will suffer a subarachnoid hemorrhage (SAH) each year (3). Sadly, the outcome from SAH is disappointing, with a high overall mortality (40-50\%) and fewer than $40 \%$ of patients returning to a functional life comparable to their premorbid condition (4). When one factors this immeasurable cost to the individual with the costs to so-ciety, in terms of loss of productivity and direct health care expenditures, it is un-derstandable why so much effort and re-sources have been allocated to finding more effective treatment measures.

\section{PATHOGENESIS AND CLASSIFICATION}


While the mechanism is not yet completely understood, it is generally felt that aneurysm formation is multifactorial, beginning with genetic factors possibly underlying developmental defects of the arterial wall, and enhanced by other vari-ables, such as hypertension, hemodynamic stress and atherosclerosis (5). Aneurysms are seen more frequently in certain medical conditions, including polycystic kidney disease, fibromuscular dysplasia and moyamoya disease as well as in conditions associated with a defect in the arterial wall, such as Marfan's syndrome, Ehlers-Danlos syndrome and pseudoxanthoma elasticum (5). Finally, acquired pathological conditions that damage the arterial wall, such as infection, neoplasm and trauma, can also lead to aneurysm formation.

Intracranial aneurysms are classified by their size, shape and site. The size is determined by the largest diam-eter of the dome of the aneurysm. Saccular aneurysms are less than $1.5 \mathrm{~cm}$, globular aneurysms are $1.5-2.5 \mathrm{~cm}$, and gi-ant aneurysms are greater than $2.5 \mathrm{~cm}$. There are four basic shapes of intracranial aneurysms, the most common being the saccular aneurysm which has a well-defined neck and dome (note that the term saccular can be used to denote both size and shape). Most idiopathic aneurysms are of this type. By contrast, the fusiform aneurysm is a diffuse enlargement without a definable neck and is commonly seen in atherosclerosis. Dissecting aneurysms arise when blood en-ters the wall of the artery, dissecting between layers, creating a cavity within the vessel wall itself. These are rare in the intracranial vasculature. Finally, a false aneurysm is an extravascular hematoma that communicates with the intravascular space. In this case, the vascular wall has been breached, and the wall of the aneurysm consists of only outer arterial layers or periarterial tissue, such as reactive connective tissue or blood clot. These are most commonly secondary to trauma or tumor (5).

With regards to site, intracranial aneurysms are usually located at the bifurcation of major arteries within the circle of Willis. The following incidences have been documented in cooperative studies: internal carotid artery 41\%; anterior communicating-anterior cerebral artery 34\%; middle cerebral artery 20\%; vertebrobasilar artery 4\%; and in other sites $1 \%$ (6).

\section{COMPLICATIONS}

A study on the natural history of unruptured intracranial aneurysms (4) suggests that the approximate an-nual incidence of aneurysmal rupture is $1.4 \%$. The incidence increases (3.6\%) in patients who are symptomatic for their aneurysm. Because of the risk of a catastrophic SAH, it is of paramount importance to recognize the early symp-toms of intracranial aneurysm. These are usually due to an-eurysm enlargement or a "warning leak" and are estimated to occur in 25 to 50 $\%$ of cases. They include new onset of headache or any mild focal neurological deficit (most com-monly eye or facial pain), visual loss or double vision. Third nerve palsies can also occur. That being said, these symp-toms may be distressingly mild, making early diagnosis dif-ficult. It is not uncommon for patients presenting with such symptoms to be sent home with instructions to rest and take analgesics, only to suffer a SAH days later. Patients suffer-ing SAH typically present with a story of a sudden onset of "the worst headache of my life", possibly with nausea 
and vomiting. Once SAH has occurred, a multitude of compli-cations may ensue, including direct cerebral injury, rebleeding, vasospasm, hydrocephalus, venous thromboembolism, diabetes insipidus, SIADH, cardiac in-jury and pulmonary edema (7).

The diagnostic work up of a patient presenting with a history suggestive of SAH includes a CT scan without enhancement, which in the first few days after SAH detects blood in the subarachnoid, subdural and intraventricular spaces, or within the brain parenchyma in over 95\% of cases $(8,9)$. This is followed by transfemoral cerebral angiography which will provide important details as to the site, shape and size of the aneurysm (Fig.1b).

\section{SURGICAL INDICATIONS AND OPTIONS COMPLICATIONS}

The most important issues to consider for surgical intervention are whether or not the aneurysm has ruptured, the accessibility of the aneurysm for repair, the configuration and size of the aneurysmal neck, the presence of a thrombus, and the relationship of the aneurysmal neck to the parent ar-tery. Aneurysmal rupture with subsequent SAH is a strong indication for surgical intervention. If the aneurysm is unruptured but symptomatic, surgery is again typically recommended, because the symptoms themselves can be disa-bling and, as mentioned, are often a warning sign of future rupture. For asymptomatic aneurysms, the indications for surgery are more controversial. It is known that the inci-dence of rupture increases with aneurysm size, and, gener-ally, if aneurysms are greater than $1 \mathrm{~cm}$, the risk of surgery is outweighed by potential to rupture in patients less than 65 years of age (10). The neurosurgeon must also consider the patient's age and general medical condition when weighing postoperative morbidity and mortality versus the natural his-tory of intracranial aneurysms and SAH.

Direct surgical clipping has been the standard defini-tive treatment for ruptured intracranial aneurysms. In this treatment, the aneurysmal neck is obliterated with a surgical clip which has the size and strength to prevent blood flow into the aneurysm, and is appropriately positioned so as to allow normal flow in the parent artery. When an aneurysm is anatomically inaccessible, surgical ligation or trapping of the offending aneurysmal segment may also be employed, but in these cases flow through the parent artery is compro-mised to seal off the aneurysm. The success of this approach depends heavily on the adequacy of collateral flow to the area. In some rarer cases of fusiform or dissecting aneu-rysms, because of the difficult nature of the anatomy, another option in treating these lesions could be wrapping or coating the aneurysm with a substance like Teflon ${ }^{\circledR}$. This technique may be used in conjunction with clipping, but is not a definitive treatment on its own.

\section{ENDOVASCULAR TECHNIQUES}

A new approach to treatment of intracranial aneu-rysms stemmed from the development of endovascular tech-niques during the 1970's $(11,12)$ and the use of transfemoral catheters with balloon tips, similar to those used for coronary angioplasty, as a therapeutic approach. 
Fig. 1(a, b) A 37 year old woman presented to the Queen Elizabeth Health Sciences Centre Emergency Room with severe headache, back and leg discomfort with some nausea and vomiting. (a) A non-enhanced CT of her head revealed a suspicious dilatation of the distal basilar artery. (b) Cerebral angiography confirmed the presence of a 9 x 6 x $9 \mathrm{~mm}$ aneurysm at the tip of the basilar artery.

This field of endovascular therapy can be separated into two broad categories: (1) occlusion of the parent artery and (2) selective occlusion of the aneurysm with detachable balloons or metallic coils (13).

\section{BALLOON TIP CATHETERS}

In some forms of aneurysm, the neck is too poorly defined and ectatic for successful surgical clipping of the an-eurysm to be possible. In these cases, occlusion of the par-ent artery is the only option for prevention of the complica-tions (i.e., aneurysmal rupture and bleeding). As mentioned, surgical ligation of the parent artery is an option here, but balloon occlusion can also be used, with favorable results when compared with surgery (5). This is always done with a trial occlusion first, to assess the patient's ability to tolerate such a disturbance in blood flow. The tolerance, once again, is related to the adequacy of collateral blood flow. Using the balloon occlusion technique for internal carotid aneurysms, parent vessel and aneurysmal occlusion was achieved in 59.1 and $40.9 \%$ of cases, respectively, in the largest North American trial (14). The associated mortality was $9.8 \%$ and the rate of stroke was $7.4 \%$. The use of balloons in the anterior circulation is felt to have some advantages over surgical ligation. First, the patient is awake during the procedure and, therefore, it is much easier to monitor neurological sta-tus. Second, the technique is carried out transfemorally, so that systemic anticoagulation therapy can be used to de-crease the chance of embolic phenomenon. Finally, the bal-loon can be detached at or just proximal to the neck of the aneurysm, reducing the possibility of the sac refilling by means of collateral circulation from the external carotid. In the posterior (vertebrobasilar) circulation, however, the anatomy is less favourable for permanent balloon occlusion and, as such, the technique is riskier and performed less commonly than in the anterior circulation. One of the major disadvantages to the balloon occlusion technique is that all balloons filled with nonsolidifying liquids will slowly de-flate over time. This may not be a problem when the goal is total parent artery occlusion because it has been observed that arterial and aneurysmal thrombosis may occur by the time the balloon has deflated.

With improvements in technology, including balloon and catheter technology, digital subtraction angiography, fluoroscopy with road-mapping capabilities and liquid so-lidifying agents, endovascular techniques have been further refined so that selective positioning of the balloon within the aneurysmal sac itself has now become possible. Investiga-tions into the use of selective balloon occlusion of intracranial aneurysms report successful occlusion rates of 77.4 to 
$91 \%(15,16)$. The technique is most effective in pa-tients with fair to good clinical status prior to the procedure. The mortality rates were as low as $1.7 \%$ in patients with a good clinical grade, but as high as $22 \%$ in patients with poor clinical grade on presentation. The major disadvantages to this approach are two-fold. First, as mentioned, balloons de-flate with time and, although thrombus may have formed, occasionally the aneurysm may recanalate. Liquid solidify-ing agents (such as hydroxyethylmethacrylate) are now usu-ally infused into the balloon just before detachment to help alleviate the problem of deflation. The second problem is $100 \%$ filling of the aneurysm with balloon. Although there are many different sizes and shapes commercially available, and more than one balloon can be used per aneurysm, it is still difficult to get the balloon(s) to completely conform to the aneurysms' dimensions. Balloons that do not achieve full occlusion of the aneurysm have also been reported to exert a "water-hammer effect" on the wall of the aneurysm from pulsating arterial blood on the balloon, which may fa-cilitate enlargement or delayed rupture of aneurysms (17).

\section{METALLIC COILS}

Another endovascular approach aimed at selective occlusion of intracranial aneurysms uses metallic coils to 'embolize’ intracranial aneurysms. This technique, which utilizes the principle of intra-aneurysmal electrothrombosis, was conceived about a decade ago. The concept was based on previous animal model studies looking at clot formation triggered by direct electrical current (for review see ref. 18). Not until recently, however, has the technology to produce microcatheters soft enough to navigate atraumatically into a distant aneurysm become available. Now, a novel device consisting of a soft detachable platinum coil soldered to a stainless steel delivery wire can be delivered directly into an intracranial aneurysm with the purpose of inducing aneurysmal embolization without affecting blood flow in the parent artery. This is accomplished via utilization of the electrochemical principles of electrothrombosis and electrolysis. Electrothrombosis refers to the induction of clot formation (i.e., a thrombus) with a direct positive electrical current. This occurs because white blood cells, red blood cells, platelets and fibrinogen are negatively charged and, if a positively charged electrode is placed in the arterial stream, these blood components will be induced to aggre- gate at the site of the electrode, facilitating clot formation. Electrolysis refers to the process that occurs when two iron electrodes connected to a source of direct current are dipped in a solution. What results is that the positive wire dissolves and the other wire recruits the migrating ferrous ions from the anode to the cathode. Electrolysis has also been studied in silver, copper, platinum and stainless steel electrodes. Platinum, being a noble metal, resists electrolysis while the other metals undergo electrolysis at variable rates. It is this phenomenon that facilitates detachment of the platinum coil from the stainless steel delivery wire within an aneurysm, because the stainless steel portion of the system will dissolve with time under the influence of the direct current being ap-plied to the platinum coil. 
The procedure was first studied in saccular aneu-rysms in swine animal models in 1989. One year later, hu-man studies were undertaken by Guido Guglielmi et al. in the Department of Radiological Sciences at the University of California Medical Centre in Los Angeles (19). All proce-dures were performed with patients awake and under sys-temic heparinization. Catheterization was transfemoral in approach and visualization was achieved via digital subtraction angiography and fluoroscopy with road-mapping capa-bilities. "Road mapping" refers to the ability to take snap shot digital angiographic images of the arterial tree of inter-est and superimpose the image over ongoing fluoroscopic visualization of efforts to reach the aneurysm, thus facilitat-ing navigation to the aneurysmal sac (see Fig. 1c).

Initially a guiding catheter is inserted transfemorally into the internal carotid or vertebral artery. Then a microcatheter is coaxially advanced through the guiding catheter into the aneurysm, with the assistance of a micro guidewire.

Fig. 1(c, d) A 37 year old woman presented to the Queen Elizabeth Health Sciences Centre Emergency Room with severe headache, back and leg discomfort with some nausea and vomiting. (c) Six GDC coils were inserted to embolize the basilar tip aneurysm. (d) Postembolization angiography demonstrating obliteration of the aneurysm with adequate flow in the parent artery and no evidence of an aneurysmal neck remnant.

The newly devised detachable platinum coil (named Guglielmi detachable coil or GDC), which is to be inserted through the microcatheter, consists of a very soft detachable platinum portion soldered to a stainless steel de-livery wire. It is introduced to the microcatheter with a spe-cial introducer and while it is in the microcatheter, it remains straight. When it is advanced beyond the tip of the microcatheter, it adopts a circular formation and folds upon itself, conforming to the shape of the aneurysm. These coils are available in various sizes, as defined by their core diam-eter, coil diameter, helical diameter and overall length. The size chosen depends on the radiologic assessment of the an-eurysm's dimensions. The platinum coil is radiopaque and allows fluoroscopic visualization while it is being positioned within the aneurysm, beyond the tip of the microcatheter (Fig 1c). When the coil is in place and the platinum/stainless steel junction zone is $3 \mathrm{~mm}$ beyond the tip of the microcatheter, a $0.5 \mathrm{~mA}, 2-\mathrm{V}$ positive direct current is applied to the stainless steel delivery wire. During the next 4 to 12 minutes a thrombus formation is induced by the reac-tion of the aforementioned blood components with the plati-num electrode and electrolysis of the stainless steel portion of the coil releases the platinum portion within the aneu-rysm. For most aneurysms, it will take more than one coil to adequately embolize the aneurysm, and these can be added as needed in similar fashion to the first. 
Since the introduction of these electrolytically de-tachable coils for the treatment of intracranial aneurysms in 1990, their effectiveness has been assessed in a prospective clinical trial involving eight interventional neuroradiology centres in the United States (20). The GDC coils were used in 403 patients presenting with SAH from 1990 to 1995. These patients were all excluded from surgical intervention because of surgical difficulty due to the size and location of the aneurysm, poor neurological grade or medical status, re-fusal of surgery or previously attempted surgical explora-tion. There were aneurysms from several different loca-tions, the most common being basilar tip aneurysms (31.5\%). For total occlusion, the technique was most effec-tive (70.8 \%) in small aneurysm with a small, well-defined neck, and less effective in larger aneurysms (35.0\%) and those without a well-defined neck (31.2\%). Approximately 8\% of patients treated in the trial required postembolization neurosurgical procedures and the overall rates of morbidity and mortality were $8.9 \%$ and $6.2 \%$, respectively. Complica-tions of the procedure included aneurysm perforation (2.7\%), cerebral clot embolization (2.5\%) and parent artery occlusion (3.0\%). The authors recommend that awareness of the potential complications and the use of heparin (up to 48 hours) followed by long-term aspirin may help reduce complication rates. The conclusions drawn from the study were that GDC embolization of intracranial aneurysms in patients presenting with SAH helped improve their natural clinical course. Even in the cases when total occlusion of the aneurysm was not achieved, the patients were still felt to of benefit from the procedure. Here embolization may serve as a short term management option to reduce the risk of rebleeding until a more definitive embolization or surgical intervention can be achieved after the acute critical period of SAH.

As an endovascular approach, coiling has several distinct advantages to balloon occlusion. The use of GDC's allows safer and easier catheterization than the flow directed detachable balloon. Also, because of the ease of electrolytic detachment of the platinum coil, they avoid potentially dangerous traction on the parent artery. GDC coiling was first performed in Halifax in August 1997 at the QE II Health Sciences Centre - New Halifax Infirmary Site (see Fig. 1).

\section{SUMMARY}

Overall, endovascular techniques for the treatment of intracranial aneurysms continue to evolve and establish their respective roles. For now, neurosurgical clipping of aneu-rysms is the only therapy to have established favorable long-term outcomes $(3,8)$ and remains the gold standard of treat-ment. Some aneurysms, however, are not suited for surgical intervention, and some patients are not ideal candidates for a neurosurgical procedure. In these cases, endovascular tech-niques offer an effective second option for treatment. GDC coiling of aneurysms is a new endovascular technique which has shown promising results (20). If these results translate into good long term 
outcomes, it can be expected to expand its role in the management of patients with intracranial aneurysm as the technology and expertise becomes more readily available. What remains to be determined in clinical trials, some of which are underway, is which patients are best suited for surgery as first line treatment and which will ben-efit more from a primary endovascular procedure.

\section{ACKNOWLEDGEMENTS}

The author would like to thank Dr. Blaise Baxter in the Department of Radiology and Diagnostic Imaging, QE II Health Sciences Centre, for critical feedback in the prepara-tion of the manuscript and for providing the case history and radiographic images for illustration.

\section{REFERENCES}

1. Atkinson JL, Sundt TM Jr, Houser OW, Whisnant JP. Angiographic frequency of anterior circulation intracranial aneu-rysms. J Neurosurg 1989;70:551-555.

2. McCormick WF, Acosta-Rua GJ. The size of intracranial saccular aneurysms: an autopsy study. J Neurosurg 1970;33:422-427.

3. Kassell NF, Drake CG. Timing of aneurysm surgery. Neurosur-gery 1982;10:514-519.

4. Juvela S, Porras M, Heiskanen O. Natural history of unruptured intracranial aneurysms: a long-term follow-up study. J Neurosurg 1993;79:174-182.

5. Meyer BM, Morita A, Puumala MR, Nichols DA. Medical and surgical management of intracranial aneurysms. Mayo Clin Proc 1995;70:153-172.

6. Sahs AL, Nibbelink DW, Torner JC, editors. Aneurysmal Subarachnoid Hemorrhage: Report of the Cooperative Study. Baltimore: Urban \& Schwarzenberg, 1981.

7. Rengachary SS, Wilkins RH. Principles of Neurosurgery. St Louis: Wolfe Publishing, 1994.

8. Kassell NF, Torner JC. The International Cooperative Study on the Timing of Aneurysmal Surgery: an update. Stroke 1984;15:566-570.

9. Adams HP, Kassell, NF, Torner JC. Usefulness of computed tomography in predicting outcome after aneurysmal subarachnoid hemorrhage: a preliminary report of the Coopera-tive Aneurysm Study. Neurology 1985;35:1263-1267.

10. Wiebers DO, Whisnant JP, Sundt TM Jr, O'Fallon WM. The sig-nificance of unruptured intracranial saccular aneurysms. J Neurosurg 1987;66:23-29. 
11. Serbinenko FA. Catheterization and occlusion of major cerebral vessels and prospects for the development of vascular neuro-surgery. Vopr Neirokhir 1971;35(No. 5):17-27.

12. Serbinenko FA. Balloon catheterization and occlusion of major cerebral vessels. J Neurosurg 1974;41:125-145.

13. Nichols DA, Meyer FB, Piepgras DG, Smith P. Endovascular treatment of intracranial aneurysms. Mayo Clin Proc 1994;69:272-285.

14. Higashida RT, Halbach VV, Dowd C, Barnwell SL, Dormandy B, Bell J, et al. Endovascular detachable balloon embolization therapy of cavernous carotid artery aneurysms: results in 87 cases. J Neurosurg 1990;72:857-863.

15. Shcheglov VI. Endovascular occlusion of saccular intracranial aneurysms: results in 617 patients. Presented at the 27th An-nual Meeting of the American Society of Neuroradiology; 1989 Mar 18-24; Orlando (FL)

16. Higashida RT, Halbach VV, Dowd C, Barnwell SL, Dormandy B, Bell J, et al. Treatment of intracranial aneurysms with preserva-tion of the parent vessel: results of percutaneous balloon embolization in 84 patients. Am J Neuroradiol 1990;11:633-640.

17. Kwan ESK, Heilman CB, Shucart WA, Klucznik RP. Enlarge-ment of basilar artery aneurysms following balloon occlusion-water- hammer effect: report of two cases. J Neurosurg 1991;75:963-968. 18. Guglielmi G, Viñuela F, Sepetka I, Macellari V. Electrothrombosis of saccular aneurysms via endovascular ap-proach. Part 1: Electrochemical basis, technique, and experi-mental results. J Neurosurg 1991;75:1-7.

19. Guglielmi G, Viñuela F, Dion J, Duckwiler G. Electrothrombosis of saccular aneurysms via endovascular approach. Part 2: Pre-liminary clinical experience. J Neurosurg 1991;75:8-14.

20. Viñuela F, Duckwiler G, Mawad M. Guglielmi detachable coil embolization of acute intracranial aneurysm: perioperative ana-tomical and clinical outcome in 403 patients. $\mathrm{J}$ Neurosurg 1997;86:475-482. 